Sugerencia de citación: Salas Arón, E. (2021). ¿Smith o Kuznets en Argentina? La matriz laboral durante la primera globalización (1870-1914). tiempo\&economía, 8(1), 11-41. https://doi.org/10.21789/24222704.1677

Vol. 8 N. ${ }^{\circ} 1$

Enero - Junio del 2021

pp. $11-41$
HISTORIA ECONÓMICA, EMPRESARIAL Y DEL PENSAMIENTO

TIEMPO \& ECONOMÍA

DOI:

https://doi.org/10.21789/24222704.1677

\title{
¿Smith o Kuznets en Argentina? La matriz laboral durante la primera globalización (1870-1914)
}

\section{Smith or Kuznets in the Growing Argentina? The Labor Matrix during the First Globalization (1870-1914)}

Emiliano Salas Arón

Consejo Nacional de Investigaciones Científicas y Técnicas (CONICET) Instituto de Historia Argentina y Americana "Dr. Emilio Ravignani", Universidad de Buenos Aires Universidad Torcuato Di Tella, Argentina https://orcid.org/0000-0002-9146-0015 emilianosalasaron@gmail.com

\section{RESUMEN}

Dentro del debate sobre la evolución de las economías en el largo plazo, dos autores clásicos, Adam Smith y Simon Kuznets, propusieron teorías contrastantes sobre la evolución del mercado de trabajo en un período de crecimiento de la economía. Smith propuso que el crecimiento se produce a partir de una especialización productiva de las economías motivada por su inserción en el comercio internacional, lo que genera una transformación en el mercado. Kuznets, en cambio, plantea que los procesos prolongados de crecimiento en las economías modernas generan una diversificación estructural de los mercados de trabajo, con una intensificación de los empleos urbanos en sectores secundarios y terciarios, los cuales involucran una mayor capacitación 
de la mano de obra. Con estos marcos teóricos, el artículo presenta un análisis de la evolución de la estructura laboral en Argentina durante la primera globalización (1870-1914). Para ello, se realizó una clasificación por sectores y destrezas de los trabajadores utilizando las secciones de ocupaciones correspondientes a los censos nacionales de 1869, 1895 y 1914. Los principales resultados demuestran que a pesar del acelerado crecimiento de la economía durante el período 1870-1895, Argentina modificó relativamente poco su estructura laboral a nivel intersectorial. No obstante, luego de 1890 se advierten una serie de transformaciones en la economía que ocasionaron cambios en el mercado de trabajo dentro de ciertos sectores, los cuales vieron modificada su composición y la intensidad de destrezas de sus trabajadores.

Palabras clave: mercado laboral, evolución sectorial, desarrollo productivo, Argentina, primera globalización, historia económica.

Códigos JEL: F16, F02, N00

\section{ABSTRACT}

In the classic theory about the evolution of economies in the long term, Adam Smith and Simon Kuznets proposed two contrasting theories about the evolution of the labor market in a period of economic growth. Smith proposed a growth in the labor markets guided by the more dynamic and productive sectors through a process of specialization led by the insertion in international commerce. Kuznets, instead, theorizes that the growing modern economies generate changes and diversification on the labor markets towards secondary and tertiary sectors and on the skills stock of the labor force. With that in mind, this work presents an analysis of the labor structure in Argentina during the first globalization period (1870-1914) through a classification of workers and the economic active population divided by sectors and skills, using the occupational compilation of the national census of 1869, 1895 and 1914. The main results show that, despite the high growth of the economy during the first part of the studied period (1870-1895), Argentina did not show big changes in the labor structure at an inter-sectoral level. However, the growth of the economy, especially after 1890, involved a series of transformations on the labor markets that modified their composition and the intensity of skills by the labor force.

Keywords: Labor markets, sectoral evolution, productive development, Argentina, first globalization, economic history.

JEL Codes: F16, F02, N00 


\section{INTRODUCCIÓN}

El debate en torno a las transformaciones generadas durante el ciclo de crecimiento económico en la primera globalización en Argentina (18801914) es uno de los que ha tenido mayor análisis en la historia económica de este país. La apertura externa y la caída de los costos de transacción para el comercio internacional provocaron una mejora de las condiciones exportadoras, al mismo tiempo que se registró una expansión notable de su stock de tierras por el avance sobre la frontera sur en la década de 1870 (Gerchunoff \& Llach, 2017, pp. 34-37; Míguez, 2012, pp. 165-167). Por otro lado, la adopción del patrón oro propició una integración virtuosa al mercado de capitales que contribuyó a un programa acelerado de obras públicas e infraestructura, así como a una expansión de los bienes públicos, como la educación o los servicios estatales (Della-Paolera \& Taylor, 2003, pp. 37-38). Adicionalmente, la caída de los costos de transporte y, en menor medida, las políticas públicas contribuyeron a un notable aumento de la oferta de mano de obra por medio de la inmigración, impulsando el crecimiento de una economía que sufrió una carestía en su fuerza de trabajo durante el siglo XIX.

Más allá de esta notable expansión económica, algunas características del crecimiento han sido discutidas. Por un lado, una serie de posturas abonaron a la idea de una expansión notable de la estructura productiva del país (ante una modernización de sus mercados y el incremento de las exportaciones) con efectos expansivos en el ingreso nacional y la productividad de los factores (Cortés-Conde, 1979; Della-Paolera \& Taylor, 2003). Sin embargo, otros autores matizaron los avances, sugiriendo que el pobre desempeño económico que sobrevino a la primera globalización en Argentina respondió a restricciones que este modelo de crecimiento impuso sobre varios ejes. Entre los principales problemas derivados del modelo agroexportador argentino se plantea la inequidad distributiva, el lento cambio estructural y la falta de diversificación exportadora (Bértola \& Ocampo, 2013; Ferrer, 2008; Llach, 2014).

En este artículo se aborda el debate acerca de la evolución de la economía argentina del período desde el análisis de los mercados de trabajo y sus transiciones. Las teorías sobre la interrelación entre el crecimiento y las estructuras laborales ha tenido una larga tradición en la historia del pensamiento económico. Dentro de los aportes teóricos clásicos, se destacan las propuestas de Simon Kuznets y Adam Smith. Estos autores, que representan 
dos interpretaciones sobre la evolución de las economías en un proceso de crecimiento prolongado, también propusieron dos teorías distintas sobre los cambios de las estructuras laborales en la larga duración. Las posiciones contrastantes de estos autores interpelan a la literatura que supone la existencia de una forma uniforme bajo la cual se producen la modernización y el cambio estructural en las economías.

Kuznets planteó que la modernización económica y el crecimiento de los países, en la larga duración, debían generar una transición de trabajadores de sectores de baja calificación a otros dinámicos y modernos, elevando así la productividad de los mismos, con el consecuente efecto expansivo sobre los ingresos (Kuznets, 1955). Dentro de la idea general, se planteó que los empleos más dinámicos estaban en los sectores secundarios y terciarios, en tanto que la modernización agrícola debía expulsar población hacia las ciudades por la tecnificación de la producción, fomentando así un proceso de cambio estructural compuesto por la modernización y la expansión de la economía urbana, la cual estaba ligada a la industrialización y al crecimiento de los servicios. El aporte de Kuznets rompió con un consenso extendido planteado inicialmente por la tradición liberal clásica.

La propuesta de Smith sobre la evolución de los mercados de trabajo es distinta, puesto que supone que la estructura del empleo se encuentra determinada por un proceso de especialización productiva de las economías sobre los sectores que tienen un proceso acelerado de acumulación de capital, sobre todo en un proceso de integración internacional (Smith, 1983, pp. 103-104, 113). Smith presenta, entonces, la hipótesis de una evolución de los mercados de trabajos que predice una movilización de la mano de obra hacia aquellos donde las economías tienen ventajas naturales o una acumulación de capital relativamente alta a medida que se va produciendo la integración a los mercados globales. En Argentina, podemos suponer que ese sector es el agropecuario, pues este se integró más eficientemente a los mercados internacionales durante la primera globalización. El contraste con la propuesta de Kuznets, en este sentido, es directo.

En síntesis, podemos indicar que el análisis de los mercados de trabajo nos provee información sobre cómo se organiza la estructura productiva a escala sectorial. Así mismo, en el análisis de la calificación de los sectores se puede indagar sobre la productividad y capacidad innovadora de estos. El artículo se divide en cinco partes. En primer lugar, se presentan los antecedentes que se encuentran disponibles en la historiografía argentina sobre la 
temática en cuestión. Luego, se describe la metodología de construcción de las tablas sociales, donde se ubicaron las diversas categorías y los sectores para su calificación. Posteriormente, se revelan los resultados de la estimación de la calificación y la evolución general de la misma durante el período. En seguida, se desarrolla un análisis de la evolución por sectores de esta matriz laboral general. Por último, se presentan una serie de conclusiones generales.

\section{EL DEBATE SOBRE LA ESTRUCTURA LABORAL Y SUS PRINCIPALES EXPONENTES}

El análisis de la estructura laboral durante la primera globalización ha tenido en Argentina algunos antecedentes relevantes. Un primer análisis, propuesto desde los estudios sociológicos, fue elaborado por Gino Germani y Jorge Garciarena. En sus trabajos se plantea que en los años que transcurren entre 1895 y 1914 hay cambios fundamentales en la distribución de los trabajos por sector, con una paulatina preponderancia del sector secundario sobre el primario (Germani \& Garciarena, 1987, p. 128). Esta hipótesis abona y fue un puntapié inicial para la hipótesis de la industrialización temprana de Argentina a partir de la crisis de 1890 y la formación de mecanismos cambiarios y arancelarios de protección para las industrias (Rocchi, 2005a).

Un segundo grupo de antecedentes, desde los estudios demográficos, fue elaborado por Zulma Recchini y Alfredo Lattes (1975) y Ernesto Kritz (1985). En estos trabajos se registran algunas tendencias sobre la matriz ocupacional entre 1869 y 1970 y se propone un análisis sobre la conformación del mercado de trabajo y el crecimiento del mismo. Se cuenta con estimaciones y un análisis detallado de las tasas de participación por sexo, origen y grupos etarios en la estructura ocupacional. En ninguno de estos dos antecedentes existe, sin embargo, un análisis profuso y detallado sobre la distribución de la mano de obra por rubros y sectores, que es la propuesta a realizar en este documento.

El análisis de los mercados de trabajo durante la primera globalización también ha sido abordado por la historia económica. Al respecto, Carlos Díaz Alejandro y Roberto Cortés Conde han planteado diversas interpretaciones sobre el proceso de creación de empleos y la distribución de la oferta laboral en los mismos. Cortés Conde argumentó que la oferta ocupacional del período tiene como característica predominante una altísima elasticidad producto de las migraciones. Estas se explican por el pull salarial 
que ofrece el mercado local, el cual promueve una numerosa migración hacia Argentina (Cortés-Conde, 1979). Esta idea fue profundizada y complejizada por trabajos posteriores (Arceo et al., 2019, p. 13, Panettieri, 1990, pp. 25-26). En los trabajados de Cortés Conde sobresale que la expansión de la frontera agrícola y el crecimiento liderado por las exportaciones ocasionaron una absorción de trabajo, donde el sector primario tiene el papel fundamental. Este autor también plantea que hay una importante expansión de los servicios públicos y las industrias de trabajo intensivos a partir de 1890, los cuales superan al sector agropecuario en absorción de empleo (Cortés-Conde, 1979, p. 199).

Cortés Conde, sin embargo, descuenta a los jornaleros de sus estimaciones sobre el peso del empleo rural en Argentina. El motivo es que existen numerosas dificultades para asignarle a este grupo de trabajadores una participación sectorial definida (Beccaria, 2006, pp. 8-9, Pianetto, 1984, p. 299); aunque ignorar a este sector puede significar un inconveniente, puesto que estos trabajadores participan de las cosechas en el sector agropecuario. Adicionalmente, el autor plantea que el mercado de trabajo en la Argentina del progreso es uno de muy bajos skills y altísima rotación, donde incluso la participación por sectores o ramas se ve alterada con mucha frecuencia (Cortés-Conde, 1979, p. 205).

A propósito de los mercados laborales en el sector agropecuario, Díaz Alejandro planteó que la subdivisión de la tierra en grandes propiedades y el tardío reparto de las mismas condicionó la ubicación de los trabajadores y la estructura salarial. Este es el motivo por el cual los migrantes llegaron al país, impulsados por la alta oferta de tierras para la agricultura, aunque terminaron consolidándose mayormente en el sector urbano ante la gran concentración de la propiedad (Díaz-Alejandro, 1970, pp. 50-51). Estas condiciones de la economía argentina fomentaron un proceso de urbanización temprano que alteró el mercado del trabajo al beneficiar la formación de sectores secundarios y terciarios, los cuales crecieron velozmente durante el período. Otros aportes han mencionado que las relaciones de producción del modelo agroexportador fomentaron un mercado laboral de baja calificación asociado a la actividad agropecuaria y una industria artesanal de bajos requerimientos de calificación (Pianetto, 1984, p. 301).

En la historiografía reciente, el debate sobre la cuestión obrera y la evolución de la economía urbana ha sido amplio. Hay antecedentes que ponderan y dan cuerpo a la idea de que existe una industria temprana que (desde 
la crisis de 1890) se volvió un sector crecientemente relevante y con un crecimiento sostenido (Rocchi, 2005b). En relación con este proceso, se identifican varios estudios donde el interés por el mercado de trabajo se asocia al proceso de formación de una clase obrera definida como tal durante el período (Suriano \& Lobato, 2004, pp. 13-15). Varios historiadores han tratado el tema de la estructura ocupacional, aunque enfocando esta temática sobre algunos subgrupos en cuestión. En este eje se han destacado los hallazgos de Fernando Devoto sobre las diferencias en la demanda de trabajo entre ciudadanos locales e inmigrantes europeos en el espacio urbano (Devoto, 2000, pp. 304-305), así como diversos autores sobre la inclusión de las mujeres dentro de este mercado laboral en formación (Allemandi, 2009, pp. 5-6; Barrancos, 2008, p. 102; Lobato, 2007, p. 247; Rocchi, 2000).

En términos generales, en casi todos los antecedentes estudiados asistimos a la idea de que luego de 1890 se dio un cambio fundamental en la estructura de los empleos causado por un proceso de crecimiento del sector secundario y terciario sobre el primario. Sobre la calificación de la oferta laboral y la utilización del capital humano, en cambio, los antecedentes han sido menos marcados. Este trabajo intenta insertarse en este debate, aportando nueva evidencia sobre los cambios sucedidos en la larga duración durante un período fuertemente expansivo en la primera globalización, tanto en la estructura laboral como en la calificación de los empleos que la economía genera.

\section{FUENTES Y METODOLOGÍA}

Para encarar el análisis del mercado de trabajo se analizaran los datos presentados para las ocupaciones en los censos nacionales llevados a cabo en 1869,1895 y $1914{ }^{1}$ En este artículo se presentan ciertos agrupamientos de los empleos por su matriz sectorial y sus niveles de calificación. Los censos presentan nóminas de ocupaciones distintas: el primero de ellos cuenta con cerca de 200 categorías, el de 1895 con alrededor de 190, mientras que el tercero cuenta con $420 .^{2}$ Estas nóminas se unificaron en 150 categorías comunes en los tres censos. Las categorías restantes se agruparon mediante un proceso de asociación a categorías similares o superpuestas.

\footnotetext{
Los volúmenes de los censos, y muy especialmente la sección de ocupaciones, que se encuentran dentro del censo general de población, fueron consultados en la Biblioteca Tornquist (Banco Central de la República Argentina).

2 Segundo Censo Nacional de Población (1895). Tomo II: Población, "Ocupaciones" en el apartado de cada una de las provincias y territorios. Tercer Censo Nacional de población (1914), Tomo IV. Talleres Gráficos Rosso, Buenos Aires, pp. 201-400.
} 
Luego, con el objetivo de realizar un ejercicio comparativo, se volvió a sintetizar estas 150 categorías en 20 grandes grupos laborales. El detalle sobre este agrupamiento de las categorías en los grupos se encuentra en el anexo 1. En algunas categorías la heterogeneidad interna no es despreciable, por lo que resulta pertinente realizar una lectura crítica de los datos aportados por las fuentes. Sin embargo, podría considerarse que estas debilidades de la fuente no impiden proponer algunas tendencias generales.

A la información de ocupaciones que presentan los censos, principalmente los de 1895 y 1914, se le realizaron algunas correcciones que perfeccionan los datos. En primer lugar, se separaron a los propietarios rurales de aquellos que aparecen como "agricultores" o "hacendados". En ambos censos de ocupaciones se registran más agricultores y hacendados que los que aparecen en la sección de propietarios agropecuarios en los censos de 1895 y 1914 , por lo que se decidió tomar el dato de propietarios de los censos agropecuarios, mientras que el resto fueron asignados a la categoría de "peones rurales". También se separó a los propietarios industriales y comerciales que aparecen en los censos respecto a los trabajadores; es decir, se separaron las categorías entre propietarios y trabajadores, utilizando los censos complementarios y datos de comercios e industrias desagregadas. ${ }^{3}$ Por último, al sector de jornaleros se le dividió en dos grupos, contemplando el sesgo hacia los trabajos temporarios agrícolas de estos sectores. Teniendo en cuenta esto, se asignó un tercio al grupo de trabajadores urbanos de baja calificación y dos tercios al grupo de trabajadores rurales de baja calificación.

Como principal fortaleza de los datos, debe tenerse en cuenta que la periodicidad de los tres censos representa cortes temporales sumamente relevantes para analizar el período. Suele considerarse a la década de 1870 como el punto de origen del proceso de crecimiento hacia afuera, acelerado hacia 1880 por la expansión territorial y las modificaciones institucionales que se dieron a partir de la federalización de Buenos Aires. Adicionalmente, los censos aportan una información integral sobre el territorio nacional, sementando las estructuras laborales de las provincias y los territorios. Contar con estas fuentes permite realizar estimaciones sobre la evolución socioeconómica de largo plazo con datos oficiales, incluso teniendo en cuenta las dificultades que podrían presentar los mismos.

3 Un procesamiento de este tipo supone, por ejemplo, que a la totalidad de los carpinteros que aparece en el censo como "trabajadores semicalificados" se restó el número total de carpinterías que figura en el censo industrial, las cuales se agrupan dentro del rubro "propietarios industriales". 


\section{LA ESTRUCTURA LABORAL DE ARGENTINA EN LA PRIMERA GLOBALIZACIÓN}

La primera globalización en Argentina fue asociada con un proceso de crecimiento liderado por las exportaciones. Esto puede afirmarse para el período 1870-1895, donde se da un proceso de crecimiento extensivo ligado a las exportaciones, especialmente ganaderas, y a una mayor conectividad de los mercados internos, donde la expansión de la red ferroviaria jugó un papel fundamental. La trayectoria que sigue entre 1895 y 1914, aunque forma parte del mismo proceso, muestra algunos contrastes importantes, con al menos dos grandes transformaciones en la estructura productiva. En primer lugar, una expansión de las redes ferroviarias en la región pampeana que facilita un cambio de eje de la producción ganadera a la agrícola, expandiendo así la productividad del sector rural. En segundo lugar, la expansión de las regiones urbanas implica un incentivo para un primer despertar industrial, que se traduce en una modernización y diversificación de la estructura productiva del país (Beccaria, 2006, p. 9; Rocchi, 2000, p. 35).

Al introducir el análisis sobre la estructura del empleo durante la primera globalización argentina lo primero que se destaca es el gran crecimiento del mercado de trabajo. La absorción de empleo en esta economía en plena expansión puede observarse en la gran atracción de inmigrantes que ingresan al país como ciudadanos o como trabajadores golondrinas durante el período. El incremento de la fuerza de trabajo en el período puede observarse en la tabla 1, que enseña las cifras de ocupación en la población mayor de 14 años en Argentina. Los datos muestran un gran aumento de la masa laboral, pasando de alrededor de 850.000 personas en 1869 a más de tres millones en 1914. Este crecimiento de la oferta de empleo fue elevado a lo largo del período, con breves picos entre 1885-1890 y 1905-1910 (Arceo et al., 2019, p. 13; Flores-Cruz, 2014, p. 6). Así mismo, los números muestran que, a nivel general, la tasa de ocupación mantiene la proporción de los empleados en Argentina entre 1895 y 1913, luego de un declive entre 1869 y 1895 . Esta expansión de la población económicamente activa, con el mantenimiento de una tasa de ocupación, coincide con el gran crecimiento del producto de la economía y de la frontera productiva a lo largo del período. 


\begin{tabular}{|c|c|c|c|c|c|c|}
\cline { 3 - 6 } Año & & Con profesión & Sin profesión & Total & \% empleados & \% desempleados \\
\hline \multirow{2}{*}{1869} & Total & 857.167 & 156.908 & 1.014 .075 & 84,53 & 15,47 \\
\hline \multirow{2}{*}{1895} & Hombres & 1.144 .950 & 176.375 & 1.321 .325 & 86,65 & 13,35 \\
& Mujeres & 503.873 & 629.556 & 1.133 .429 & 44,46 & 55,54 \\
& Total & 1.648 .823 & 805.931 & 2.454 .754 & 67,17 & 32,83 \\
\hline \multirow{2}{*}{1914} & Hombres & 3.200 .001 & 257.671 & 3.457 .672 & 92,55 & 7,45 \\
& Mujeres & 735.836 & 1.535 .990 & 2.271 .826 & 32,39 & 67,61 \\
& Total & 3.935 .837 & 1.793 .661 & 5.729 .498 & 68,69 & 31,31 \\
\hline
\end{tabular}

Tabla 1.

Empleo y desempleo de la población mayor de 14 años en Argentina (1870-1914)

Fuente: elaboración propia con base en los censos nacionales de 1869,1895 y 1914
Otro elemento destacable es el aumento del peso demográfico de los hombres con respecto al de las mujeres en el proceso de la gran migración, que en la tabla 1 se ve a partir de 1895. Los datos censales revelan que entre los hombres las tasas de empleo entre 1870 y 1913 aumentan, llegando a un estado de parcial pleno empleo en 1914. ¿A qué se debe la existencia de una proporción tan importante de población sin trabajo? Como se observa en la tabla 1, esto es un efecto de la marcada reducción del nivel de empleo femenino, hecho que ha sido destacado por numerosos antecedentes historiográficos del período (Allemandi, 2009; Cortés-Conde, 1979, p. 197; Kritz, 1985, pp. 45-50; Recchini \& Lattes, 1975, p. 153).

La pregunta sobre por qué las mujeres son desplazadas del mercado laboral es un tema en sí mismo y merece una discusión más extendida. No obstante, podemos plantear tentativamente cuatro hipótesis por las cuales esto podría ocurrir: (i) porque los sectores más intensivamente demandantes de mano de obra femenina, entre los cuales destacan las hilanderías y las tejedurías, fueron especialmente afectados por la apertura externa y el crecimiento de la oferta de importaciones en la globalización (Kritz, 1985, pp. 59-61); (ii) porque Argentina entró en una instancia de desarrollo superior impulsada por su crecimiento económico, con efectos expansivos sobre el salario real y a nivel familiar, lo que permite una ponderación superior de otros bienes para los hijos -educativos, sanitarios, sociales-, cuya provisión generó una salida de las madres de familia y los niños del mercado laboral (De-Vries, 2008, pp. 190-195, 203-205); (iii) porque en 1870 existe una "artificial" inclusividad de las mujeres en el mercado laboral ante la gran carestía de trabajadores que generan las levas de hombres para los combates de la "Guerra de la Triple Alianza"; (iv) porque lo que se computa en 1869 
como empleo femenino deja de serlo por los censistas en instancias posteriores, generando una toma de información distinta que tiende a debilitar la falibilidad de la comparación (Barrancos, 2008, p. 102).

Al pensar en la estructura por niveles de skills, podríamos suponer que en una economía que crece y se diversifica, como la del período, la demanda de trabajos calificados aumentará por la misma evolución de la estructura productiva, como predice la hipótesis de Kuznets. Esta suposición se refuerza si pensamos en un modelo predominantemente agrícola que inicia un proceso de urbanización y diversificación productiva tempranamente, en términos relativos a otros países de la región (Bértola \& Ocampo, 2013, pp. 155-162; Recchini \& Lattes, 1975, p. 115). Además, la ampliación de las redes ferroviarias y la conectividad de los mercados interiores facilitó la integración regional y sectorial de los trabajadores, contribuyendo así a una veloz relocalización de estos en los sectores más dinámicos.

En la tabla 2 podemos observar qué ocurre con la dinámica sectorial del empleo y las calificaciones del mismo. Frente a un crecimiento tan acelerado, como el que vimos en la tabla 1, es razonable pensar que todos los sectores crecen en términos absolutos. Sin embargo, estos difieren en su participación relativa y puede observarse que el principal damnificado en el largo plazo es el sector productor de materia prima, fuertemente asociado a la economía agroexportadora. Mientras que entre 1870 y 1895 no parece haber grandes cambios en el peso del sector, entre 1895 y 1914 el total de los trabajadores rurales tiene un declive, evidenciado en la caída de la participación de los trabajadores rurales de 35,1 \% en 1895 a 27,6 \% en 1914.

En cuanto a la calificación de los trabajadores en el sector agropecuario, los datos sobre la estructura son menos claros. A pesar de esto, puede advertirse una persistencia muy importante en la participación general de los trabajadores no calificados rurales, entre los que predominan los jornaleros. Esta tendencia a generar empleos temporales, de alta volatilidad, que ya era importante en 1870, parece haberse sostenido a pesar de la transformación económica del campo argentino hacia la actividad agrícola desde la ganadería extensiva hacia 1914. 


\begin{tabular}{|c|c|c|c|c|c|c|}
\hline & $\begin{array}{c}\text { Perceptores } \\
1870\end{array}$ & $\begin{array}{c}\text { Perceptores } \\
1895\end{array}$ & $\begin{array}{c}\text { Perceptores } \\
1914\end{array}$ & $\begin{array}{c}\% \\
1870\end{array}$ & $\begin{array}{c}\% \\
1895\end{array}$ & $\begin{array}{c}\% \\
1914\end{array}$ \\
\hline $\begin{array}{c}\text { Trabajadores urbanos no } \\
\text { calificados }\end{array}$ & 277.989 & 458.029 & 781.040 & 32,7 & 24,8 & 23,3 \\
\hline $\begin{array}{l}\text { Trabajadores urbanos } \\
\text { semicalificados }\end{array}$ & 36.520 & 112.727 & 384.649 & 4,3 & 6,1 & 11,5 \\
\hline $\begin{array}{l}\text { Trabajadores rurales } \\
\text { no calificados }\end{array}$ & 177.959 & 258.582 & 578.658 & 20,9 & 14,0 & 17,2 \\
\hline $\begin{array}{l}\text { Trabajadores rurales } \\
\text { semicalificados }\end{array}$ & 122.785 & 390.789 & 328.610 & 14,4 & 21,1 & 9,8 \\
\hline Empleados & 12.846 & 108.617 & 339.570 & 1,5 & 5,9 & 10,1 \\
\hline $\begin{array}{c}\text { Productores } \\
\text { agropecuarios medios }\end{array}$ & 16.615 & 184.928 & 273.651 & 2,0 & 10,0 & 8,2 \\
\hline $\begin{array}{c}\text { Propietarios } \\
\text { agropecuarios } \\
\text { grandes }\end{array}$ & 52.637 & 54.029 & 76.484 & 6,2 & 2,9 & 2,3 \\
\hline Profesionales & 3.161 & 12.859 & 35.811 & 0,4 & 0,7 & 1,1 \\
\hline $\begin{array}{l}\text { Profesionales } \\
\text { educativos }\end{array}$ & 7.461 & 14.765 & 53.866 & 0,9 & 0,8 & 1,6 \\
\hline $\begin{array}{l}\text { Propietarios } \\
\text { industriales }\end{array}$ & 14.699 & 23.738 & 45.925 & 1,7 & 1,3 & 1,4 \\
\hline $\begin{array}{l}\text { Propietarios } \\
\text { comerciales }\end{array}$ & 20.000 & 49.815 & 93.351 & 2,4 & 2,7 & 2,8 \\
\hline Militares & 22.132 & 30.711 & 25.935 & 2,6 & 1,7 & 0,8 \\
\hline $\begin{array}{l}\text { Trabajadores } \\
\text { domésticos }\end{array}$ & 83.448 & 123.187 & 211.309 & 9,8 & 6,7 & 6,3 \\
\hline Empleados públicos & 1.065 & 23.913 & 111.371 & 0,1 & 1,3 & 3,3 \\
\hline Religiosos & 1.449 & 2.994 & 5.622 & 0,2 & 0,2 & 0,2 \\
\hline Total & 850.496 & 1.849 .683 & 3.354 .852 & 100 & 100 & 100 \\
\hline
\end{tabular}

Tabla 2.

Matriz ocupacional por sector y calificación en Argentina (1870-1914)
Fuente: elaboración propia con base en los censos nacionales de 1869,1895 y 1914.

Al analizar la evolución del peso de los propietarios sobre la estructura de empleos encontramos otra característica relevante. Esta se asocia a la relevancia de la participación de los agricultores medios (cuyas extensiones de tierra no superan 1.000 hectáreas) en contraste con los hacendados, sobre todo en los censos de 1895 y $1914 .{ }^{4}$ El declive de la estancia,

4 Para subdividir los propietarios por extensión para 1895 se utilizaron los informes de investigación parlamentaria de agricultura, ganadería e industrias derivadas (1898) y sus anexos disponibles para varias provincias o territorios (Buenos Aires, Santa Fe, Tucumán, Santiago del Estero, Chaco y Formosa). Para 1914, en cambio, se utilizaron los datos censales sobre el tamaño de las propiedades agrícolas y ganaderas. 
en un proceso de fragmentación propietaria y transformación productiva, se ve en la evolución demográfica de estos sectores propietarios. La toma de datos es defectuosa por la naturaleza cambiante del empleo de los jornaleros y la ausencia de estadísticas económicas complementarias para el censo de 1869, por lo cual no pueden tomarse evidencias concluyentes de las cifras estimadas.

En cuanto a la economía urbana, se destaca de la muestra la alta participación de los sectores urbanos no calificados en 1869, posiblemente asociada a la vigencia de las industrias artesanales locales en el interior. Este sector, evidentemente, sufre los coletazos del ingreso de las importaciones y la baja competitividad de sus producciones a partir de la expansión ferroviaria y la integración del mercado nacional, situación que se evidencia en la tendencia declinante de esta categoría hacia 1895. En 1914 se observa una reversión de este patrón, con una vuelta del crecimiento del empleo urbano, ahora asociado a estructuras industriales que se expanden velozmente desde el cambio de siglo (Gerchunoff \& Llach, 2017, p. 46). Además del crecimiento global del empleo urbano entre 1895 y 1913, también se observa un crecimiento de la calificación de este. Igualmente, se podría identificar una tendencia que supone una ampliación y modernización productiva del sector manufacturero.

Al igual que los sectores urbanos obreros e industriales, también se advierte un crecimiento importante del sector comercial (propietarios y empleados) y, sobre todo, en la administración pública. El crecimiento de los sectores comerciales, al igual que las industrias, puede explicarse por el crecimiento demográfico, el proceso de urbanización y el avance de la integración regional, que suponen un ensanchamiento de los mercados internos. La administración pública también tiene un crecimiento relevante en el período. Independientemente del retroceso del peso de las fuerzas armadas en la estructura general luego de la Guerra de la Triple Alianza (1865-1870), se cuenta con una expansión de la burocracia y del sector educativo durante el período. Los cuatro rubros de los trabajadores públicos (empleados de administración, militares, educadores y religiosos) pasaron de representar 3,8\% del total de los trabajadores en 1870 a 6 \% en 1914.

Un sector que tiende a perder participación a lo largo del período es el del personal de servicio doméstico y las labores del hogar. Hemos visto que esta economía crece mientras aparta a las mujeres de su mercado laboral. Es posible que esta salida haya generado un efecto depresor sobre el sector de 
servicio doméstico, puesto que al volver las trabajadoras al empleo doméstico las familias dejan de demandar en el mercado bienes y servicios que pasan a realizarse en el hogar. Este proceso de retorno a la dinámica tradicional en la organización del hogar y los trabajos de las familias es propio de las sociedades industriales del siglo XIX donde se produce un aumento del ingreso salarial (De-Vries, 2008, pp. 190-195). Sin embargo, como se mencionó, también es probable que en la clasificación del empleo femenino en los censos de 1895 y 1914 haya una subestimación de los trabajos domésticos por parte de los propios censistas (Barrancos, 2012), aunque la tendencia general muestra que el sector no fue altamente dinámico dentro de la estructura.

Sobresale también la baja capacidad de esta economía, en plena expansión, de generar incrementos notables de la mano de obra empleada en sectores profesionales. Estos son los empleos con la más alta escolarización y capacidad de innovación, e involucran a los trabajadores de letras y ciencias, bellas artes, jurisprudencia y profesiones sanitarias. En 1870, la participación de estos sectores es menor a 0,5\% del total, representando una cifra baja dentro de la estructura general. En 1914, luego de varios años de modernización económica, el sector profesional independiente solo ocupaba poco más de $1 \%$ de los trabajadores. Es decir, aunque es un sector en expansión, en 1913 sigue ocupando un lugar marginal en la estructura laboral del país.

\section{EL DESEMPEÑO SECTORIAL DE LOS MERCADOS LABORALES}

A continuación, se propone un análisis de la estructura laboral por sector. En la tabla 3 presentan los datos de la evolución de los sectores económicos reducidos por cantidad de empleados. El detalle de selección de empleos por sector se encuentra en el anexo 2. En términos generales, se destaca el crecimiento uniforme en los subperíodos 1869-1895 y 1895 y 1914, con un aumento anual de entre 2,5 y $3 \%$ de la masa de trabajadores en la economía argentina.

A nivel sectorial, la composición se modifica y muestra algunas cuestiones interesantes. Se ve cómo el peso del sector agropecuario y terciario crecen por encima de la media en el primer período de la muestra (18701895), mientras que el sector secundario crece relativamente poco. En el segundo período la situación es inversa, con un aumento superlativo del peso 
ocupacional del sector industrial sobre el agropecuario y comercial. Los otros sectores desagregados, correspondientes a los profesionales y al sector público, crecen a una tasa muy superior a la media nacional, lo que se debe en gran medida al nivel reducido de participación que estos sectores tienen a lo largo del período en la muestra. En la parte subsiguiente del texto se analizarán los cambios en cada uno de estos sectores por separado.

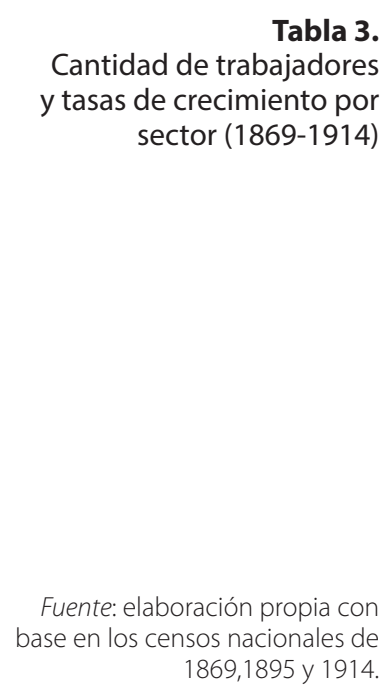

\begin{tabular}{|c|c|c|c|c|c|}
\hline Sector & $\mathbf{1 8 6 9}$ & $\mathbf{1 8 9 5}$ & $\mathbf{1 9 1 4}$ & $\begin{array}{c}\text { Tasa de crecimiento } \\
\text { promedio anual } \\
\text { (1869-1895) }\end{array}$ & $\begin{array}{c}\text { Tasa de crecimiento } \\
\text { promedio anual } \\
\text { (1895-1914) }\end{array}$ \\
\hline Agropecuario & 353.133 & 766.084 & 1.272 .815 & 3,1 & 1,9 \\
\hline Industrial & 280.773 & 460.445 & 966.446 & 1,9 & 3,1 \\
\hline Terciario & 197.537 & 533.082 & 877.405 & 3,9 & 1,9 \\
\hline Público y sanitario & 15.430 & 72.499 & 208.758 & 6,1 & 5,1 \\
\hline $\begin{array}{c}\text { Profesionales } \\
\text { y estudiantes }\end{array}$ & 6.170 & 25.857 & 93.035 & 5,7 & $\mathbf{2 , 4}$ \\
\hline Total & $\mathbf{8 5 3 . 0 4 3}$ & $\mathbf{1 . 8 5 7 . 9 6 7}$ & $\mathbf{3 . 4 1 8 . 4 5 9}$ & $\mathbf{3 , 1}$ & \\
\hline
\end{tabular}

\section{Sector agropecuario}

A lo largo de la primera globalización la trayectoria del sector agrícola muestra dos cambios relevantes. En primer lugar, sobre todo luego de la década de 1870, se produce la expansión de la frontera sur pampeana. Esto significa, en el corto plazo, un gran incremento en el stock de recursos naturales. El segundo gran cambio se asocia al proceso de cambio productivo del campo, cuya estructura se traslada desde la ganadería hacia la agricultura luego de una expansión del precio de los granos en la década de 1890 y de la densificación de la estructura ferroviaria en la Pampa húmeda (Barsky \& Gelman, 2001, pp. 160-165).

En este marco, la ampliación de la frontera agrícola moderó la presión sobre la tierra y multiplicó la cantidad de propietarios con acceso a los nuevos territorios ocupados (Sábato, 1989). Una objeción posible podría indicar que el reparto de la tierra de frontera no se realiza en forma democrática, sino más bien concentrada. Sin embargo, sin un stock de trabajadores abundantes el salario se valorizará lo suficiente como para obtener acceso a la tierra en un plazo temporal reducido. Esta idea se refuerza si tenemos en 
cuenta que, a pesar de la movilización forzada de la mano de obra en la Pampa realizada por medio de dispositivos institucionales del Gobierno Nacional (Suriano-Lobato, 2014, p. 15), la represión sobre la demanda salarial parece haber sido moderada, permaneciendo en niveles elevados durante el período (Cortés-Conde, 1979).

Entre 1900 y 1913 se comienza a advertir gradualmente el cierre de la frontera agrícola pampeana. Con esta tendencia, la abundancia de tierras disponibles se reduce, puesto que la oferta de trabajo comienza a incrementar más velozmente que la oferta de tierras. Asociado al agotamiento de frontera, algunos autores han contemplado un viraje institucional que supone un retraso de los proyectos colonizadores y la expansión de la gran estancia en las regiones de asentamiento reciente (Díaz-Alejandro, 1970, p. 52; Barsky \& Gelman, 2001, pp. 200-204). No obstante, el cambio productivo hacia la agricultura del espacio pampeano tiene también impactos en la matriz propietaria. En una estructura agraria agrícola la pequeña y mediana propiedad tiene ventajas respecto a la gran estancia, cuyas economías de escala favorecen los sistemas de explotación de la ganadería de cría. En este marco, al igual que en el contexto de apertura de la frontera sur, las condiciones productivas del campo impulsan una subdivisión de la gran estancia pampeana.

Frente a este escenario, podemos ver que a lo largo del período se reproducen condiciones para un crecimiento de los propietarios pequeños y medianos, mientras que el crecimiento de los grandes propietarios es moderado y condicionado por las estructuras laborales y productivas. Así mismo, el crecimiento de la actividad agrícola impulsa una importante modernización del campo a partir de la introducción de técnicas de cultivo y la ampliación del capital fijo en la producción (Volkind, 2015, pp. 249-252, 349-353). Sobre el perfil de los trabajadores rurales, por los defectos de las fuentes, no podemos obtener conclusiones robustas, excepto la mencionada persistencia de los trabajos estacionales de alta movilización y baja calificación a lo largo del período.

Posiblemente existan tendencias distintas entre regiones y una objeción potencial es que este cálculo a nivel nacional ignora la heterogeneidad a nivel regional. Debe tenerse presente, en trabajos futuros, que las tendencias productivas y laborales de los sectores rurales de la región pampeana podría estar ocultando un proceso de concentración territorial en el interior del país, cuyos rasgos no pueden verse en la estimación nacional. 


\section{Sector industrial/manufacturero}

A partir de los datos en la tabla 3, se propuso que puede encontrarse una tendencia hacia la ampliación y tecnificación de los sectores secundarios a partir de la década de 1890. En cuanto a la estructura interna de trabajos del sector manufacturero, la tabla 4 propone una división de los trabajos dentro la estructura industrial para los tres censos de los que se dispone de información. En los resultados podemos ver una muestra de la modernización industrial, sobre todo en la segunda parte del período. En la primera mitad, que corre entre 1870 y 1895 , el crecimiento del sector industrial es moderado en términos globales, aunque puede observarse un principio de modernización al analizar las participaciones sectoriales, ya que la cantidad de trabajadores no calificados crece mucho menos que la de los obreros calificados y propietarios.

Tabla 4.

Matriz de trabajadores de la industria y la construcción por calificación en Argentina (1870-1914)

Nota: la categoría "No calificados" incluye algunos cuentapropistas.

Fuente: elaboración propia con base en los censos nacionales de 1869,1895 y 1914.

\begin{tabular}{|c|c|c|c|c|c|c|}
\hline $\begin{array}{c}\text { Tipo de } \\
\text { trabajadores }\end{array}$ & 1870 & 1895 & 1914 & $\begin{array}{c}\text { Participación } \\
1870\end{array}$ & $\begin{array}{c}\text { Participación } \\
1895\end{array}$ & $\begin{array}{c}\text { Participación } \\
1914\end{array}$ \\
\hline No calificados & 240.047 & 356.011 & 629.530 & 85,5 & 75,7 & 65,1 \\
\hline $\begin{array}{c}\text { Semicalificadosy } \\
\text { calificados }\end{array}$ & 37.162 & 90.696 & 290.989 & 13,2 & 19,3 & 30,1 \\
\hline Propietarios & 3.564 & 23.738 & 45.925 & 1,3 & 5,0 & 4,8 \\
\hline Total & 280.773 & 470.445 & 966.444 & 100 & 100 & 100 \\
\hline
\end{tabular}

Este cambio estructural tiende a profundizar luego de 1895, cuando el incremento del peso del sector industrial en la matriz laboral se duplica, superando el crecimiento del total de ocupaciones. Visto desde la expansión de los mercados laborales, se trata de un período de importante expansión de la actividad industrial de Argentina que supone, aparte, un incremento en la intensidad de capital de las industrias ${ }^{5}$ y su demanda de trabajos calificados, tendencia que puede identificarse en los datos presentados en la tabla 4.

Esta muestra tiene algunas críticas posibles. En primer lugar, ante las limitaciones de las fuentes, se optó por realizar una síntesis muy general en

5 A partir del análisis de los censos industriales, se advierte que en el período la capitalización de las industrias (en capital declarado en pesos oro) crece mucho más aceleradamente que la cantidad de propietarios. De esta manera, puede advertirse un principio de concentración de capital industrial y comercial. 
la categoría de "trabajadores industriales", colocándolos en el sector semicalificado. En segundo lugar, es posible que la selección entre trabajadores no calificados y calificados, presentada en el anexo 1, sea imprecisa y ubique a sectores muy amplios en una sola categoría. En tercer lugar, es posible que exista en los datos una subestimación de los propietarios en la actividad industrial, al tomar a muchos cuentapropistas como trabajadores no calificados, quienes podrían ser también propietarios de un pequeño stock de capital.

No obstante lo anterior, aun teniendo en cuenta estas críticas posibles, puede advertirse una tendencia estable a lo largo del período. La misma supone que a lo largo del ciclo hay una recategorización de los trabajadores y un incremento de la calificación de los mismos. No podría decirse lo mismo de la evolución general del sector industrial, que crece lentamente hasta 1895 y muy aceleradamente después de este año.

\section{Sector terciario}

Al hablar del rubro terciario se presupone que la modernización económica genera un crecimiento muy notorio en el sector mercantil, comercial y bancario en el marco de la primera globalización. Efectivamente, en la tabla 3 podemos ver cómo se expresa esta evolución. Se ve que mientras entre 1870 y 1895 el sector terciario crece más que el promedio nacional, este aumenta ligeramente menos que el total en el segundo período. En líneas generales, podemos encontrar que la dinámica del sector es muy similar a la tendencia nacional, acompañando el ciclo general de la economía.

Al adentrarse en la estructura interna del sector pueden encontrarse algunas tendencias generales particulares. Los datos sobre la participación de los empleados y los propietarios en el sector puede verse en la tabla 5. La información disponible muestra que existe una disminución de los propietarios a lo largo de la muestra, y un incremento de los empleados y dependientes.

Lo que muestra más fluctuaciones, sin embargo, es el análisis por rubros dentro del sector terciario. Los datos de la tabla 6 indican que la participación del comercio y las finanzas es muy dinámica y crece en forma sostenida a lo largo del período casi linealmente. El rubro de transportes, en cambio, mantiene una estabilidad muy notable. La modernización de este sector es una de los más notorias por el reemplazo de los medios tradicionales, de tracción a sangre, por los modernos, de energía a vapor. Esto, empero, no 
impacta sobre la participación de los empleados en la totalidad del sector, lo que equivale a decir que se da un efecto de sustitución, donde los nuevos transportes logran equilibrar la oferta de trabajadores de los viejos. El sector que muestra una caída, como se dijo anteriormente, es el de los servicios domésticos. Por último, podemos plantear que los rentistas incrementan su participación, aunque permanecen teniendo una posición marginal en la estructura general del sector.

Tabla 5. Participación de empleados y propietarios en la actividad comercial y de servicios

Fuente: elaboración propia con base en los censos nacionales de 1869,1895 y 1914.

Tabla 6. Segmentación por rubros del sector terciario en varias categorías

Fuente: elaboración propia con base en los censos nacionales de 1869,1895 y 1914.

\begin{tabular}{|c|c|c|c|c|c|c|}
\hline Por propiedad & $\mathbf{1 8 7 0}$ & $\mathbf{1 8 9 5}$ & $\mathbf{1 9 1 4}$ & $\begin{array}{c}\text { Participación } \\
\mathbf{1 8 7 0}\end{array}$ & $\begin{array}{c}\text { Participación } \\
\mathbf{1 8 9 5}\end{array}$ & $\begin{array}{c}\text { Participación } \\
\mathbf{1 9 1 4}\end{array}$ \\
\hline $\begin{array}{c}\text { Empleados y } \\
\text { dependientes }\end{array}$ & $\mathbf{1 6 0 . 4 8 6}$ & $\mathbf{3 6 1 . 0 2 9}$ & $\mathbf{7 7 0 . 2 0 9}$ & 81,2 & 86,7 & 89,2 \\
\hline Propietarios & 37.051 & 55.477 & 93.320 & 18,8 & 13,3 & 10,8 \\
\hline Total & $\mathbf{1 9 7 . 5 3 7}$ & $\mathbf{4 1 6 . 5 0 6}$ & $\mathbf{8 6 3 . 5 2 9}$ & $\mathbf{1 0 0}$ & $\mathbf{1 0 0}$ & $\mathbf{1 0 0}$ \\
\hline
\end{tabular}

\begin{tabular}{|c|c|c|c|c|c|c|}
\hline Por rubro & 1870 & 1895 & 1914 & $\begin{array}{c}\text { Participación } \\
1870\end{array}$ & $\begin{array}{c}\text { Participación } \\
1895\end{array}$ & $\begin{array}{c}\text { Participación } \\
1914\end{array}$ \\
\hline $\begin{array}{c}\text { Comercio y } \\
\text { finanzas }\end{array}$ & 43.793 & 143.712 & 383.655 & 22,2 & 31,4 & 43,6 \\
\hline Transporte & 27.656 & 63.053 & 110.496 & 14,0 & 13,8 & 12,6 \\
\hline $\begin{array}{c}\text { Servicios } \\
\text { domésticos }\end{array}$ & 120.172 & 222.742 & 322.393 & 60,8 & 48,6 & 36,6 \\
\hline Rentistas & 5.916 & 28.445 & 63.468 & 3,0 & 6,2 & 7,2 \\
\hline Total & 197.537 & 457.952 & 880.012 & 100 & 100 & 100 \\
\hline
\end{tabular}

\section{El perfil del estado: crecimiento moderado y tendencias "civilistas"}

Asociado al sector terciario también se estimaron cuestiones en torno al empleo en el estado, pensándolo como un proveedor de servicios y bienes públicos. Una mirada sobre su peso laboral en la estructura demográfica es un indicador del crecimiento en la cobertura y calidad de los mismos. En la tabla 3 pudimos observar que es un sector en expansión durante el período. Antes de presentar los datos, hay una serie de aclaraciones respecto a los cálculos que se presentan.

En primer lugar, hay que tener en cuenta la posible alteración de los números del escalafón militar en 1870, causadas por la Guerra del Paraguay. Estos números son relativamente altos respecto a los otros años en cuestión, 
pero no podemos dejar de observar que es posible que aun en el censo de 1869 estén subrepresentados, debido a las dificultades propias de censar a los soldados en el frente de batalla. En segundo lugar, es importante remarcar las restricciones que presentan las fuentes para dar información certera sobre la administración pública. En el caso de 1869 ni siquiera se encuentra a la misma separada de otros rubros, como ocurre en 1895 y 1914. Sin embargo, en estos dos últimos censos no existe ningún tipo de desagregación sobre los empleados del sector público y de otros sectores que dependen del estado, que pueden haber sido contabilizados en otros rubros laborales, como los militares, policías, médicos o docentes.

Teniendo en cuenta las dificultades de las fuentes, y para intentar ampliar la información sobre el peso del estado en la matriz laboral, se realizaron tres estimaciones de la misma. En la primera, se toman solo los empleados de la administración, los encargados de la defensa y la seguridad y los trabajadores del sector educativo. En la segunda estimación sumamos al sector religioso en la estimación de empleados públicos. Por último, en la tercera estimación se agregó al sector sanitario. Suponemos que este último sector tiene solo una parcial dependencia del estado, ya que gran parte de los profesionales podrían realizar sus actividades en el sector privado. Con las aclaraciones correspondientes, podemos analizar los datos presentados en la tabla 7.

\begin{tabular}{|c|c|c|c|c|c|c|}
\cline { 2 - 7 } \multicolumn{1}{c|}{} & 1870 & 1895 & 1914 & $\begin{array}{c}\text { Participación } \\
1870\end{array}$ & $\begin{array}{c}\text { Participación } \\
1895\end{array}$ & $\begin{array}{c}\text { Participación } \\
1914\end{array}$ \\
\hline Trabajadores estatales & 12.466 & 45.338 & 160.767 & 1,5 & 2,8 & 4,9 \\
\hline $\begin{array}{c}\text { Trabajadores estatales } \\
\text { (incluyendo trabajado- } \\
\text { res religiosos) }\end{array}$ & 13.915 & 48.253 & 166.395 & 1,6 & 3,0 & 5,0 \\
\hline $\begin{array}{c}\text { Trabajadores estatales } \\
\text { (incluyendo trabajado- } \\
\text { res religiosos) y sector } \\
\text { sanitario }\end{array}$ & 15.430 & 53.095 & 181.148 & 1,8 & 3,3 & 5,5 \\
\hline $\begin{array}{c}\text { Total de trabajadores } \\
\text { del país }\end{array}$ & 854.880 & 1.603 .262 & 3.301 .600 & 100 & 100 & 100 \\
\hline
\end{tabular}

Los resultados muestran que el crecimiento de las tres estimaciones son similares y que el estado, si bien no muestra un peso notable en ningún momento, tiene un crecimiento importante en relación con la matriz general. ¿Qué explica que el estado crezca tanto en su tamaño absoluto y relativo respecto al total? Indudablemente, la respuesta se encuentra asociada a la mencionada ampliación de la oferta de bienes públicos.
Tabla 7.

Matriz de los trabajadores del estado (cantidad y participación sobre el total de los trabajadores activos en Argentina)

Fuente: elaboración propia con base en los censos nacionales de 1869,1895 y 1914. 
En la tabla 8 podemos ver un cálculo desagregado del rubro "trabajadores del estado" entre sus principales funciones (administración, defensa y educación). Lo más destacable, al contrario de lo que supondríamos al plantear la centralidad que tiene la expansión educativa, es que dicho sector aumenta moderadamente su participación relativa entre 1870 y 1914. A lo largo del período también sobresale la pérdida de posiciones relativas del sector defensa. Hacia 1870, los cálculos podrían estar alterados por la guerra de la Triple Alianza, pero el hecho de que el rubro militar siga perdiendo posiciones entre 1895 y 1914 revela que es un sector que ha quedado parcialmente desplazado en la estructura estatal argentina. Los datos evidencian una importante modernización de la estructura estatal con la caída del sector militar y la emergencia de los de administración, seguridad y educación pública.

Tabla 8.

Rubros de trabajadores del estado desagregados y participación en el total de los mismos

Fuente: elaboración propia con base en los censos nacionales de 1869,1895 y 1914.

\begin{tabular}{|c|c|c|c|c|c|c|}
\hline Sector & $\mathbf{1 8 7 0}$ & $\mathbf{1 8 9 5}$ & $\mathbf{1 9 1 4}$ & $\begin{array}{c}\text { Participación } \\
\mathbf{1 8 7 0}\end{array}$ & $\begin{array}{c}\text { Participación } \\
\mathbf{1 8 9 5}\end{array}$ & $\begin{array}{c}\text { Participación } \\
\mathbf{1 9 1 4}\end{array}$ \\
\hline $\begin{array}{c}\text { Administración y } \\
\text { orden público }\end{array}$ & 1.065 & 23.934 & 111.213 & 8,5 & 50,8 & 69,2 \\
\hline Educación & 2.310 & 10.063 & 39.996 & 18,5 & 21,4 & 24,9 \\
\hline Defensa & 9.091 & 13.072 & 9.558 & 72,9 & 27,8 & 5,9 \\
\hline Total & $\mathbf{1 2 . 4 6 6}$ & $\mathbf{4 7 . 0 6 9}$ & $\mathbf{1 6 0 . 7 6 7}$ & $\mathbf{1 0 0}$ & $\mathbf{1 0 0}$ & $\mathbf{1 0 0}$ \\
\hline
\end{tabular}

Sin embargo, no se debe exagerar el perfil "civilista" del estado hacia fines del período. Se registra un declive del total de los profesionales de defensa en 1914, que no supone una caída tan abrupta del gasto fiscal en este rubro, ya que desde 1901 existe en Argentina la Ley de Servicio Militar Obligatorio, que reemplaza la nómina de soldados por la de jóvenes conscriptos cuyos servicios son prestados de manera obligatoria. Debe tenerse presente que este sector no integra a los policías, quienes se encuentran en el rubro administrativo por formar parte del cuerpo de empleados de las provincias.

Podemos concluir, entonces, que el sector público más pujante es el de administración y orden público. Los números de este se revelan muy bajos en 1870, por lo que es posible asumir que esto se debe a un posible defecto de la fuente censal, que subestima la cantidad de los empleados públicos a nivel provincial, al juzgar las grandes diferencias numéricas que 
muestra la Capital Federal con el resto de las jurisdicciones. Pese a esto, el hecho de que dicha administración siga ganando posiciones luego de 1895 da fuerza a la hipótesis que plantea una tendencia ascendente del sector, que incluye tanto a empleados de dependencias como a policías provinciales.

La reducida evolución del stock de capital humano: los profesionales y la educación superior

Este apartado se enfoca en los avances en términos de capital humano y productividad; aspectos relevantes para los estudios sobre el desarrollo económico de los países. A propósito de esto, se observa, en primer lugar, el stock de profesionales con educación superior en la estructura ocupacional. Luego, se presenta la evolución de la población mayor a 14 años que aún se encuentra en condición de "estudiantes". ¿Por qué son relevantes estos cálculos? El primero significa un indicador de la capacidad de la economía de fomentar sectores productivos y científicos de más alta capitalización y capacidad de innovación. El segundo cálculo, sobre los estudiantes, a diferencia de los profesionales, es un cálculo aproximado de la evolución futura del rubro profesional.

En la tabla 3 hemos podido observar que la categoría de "profesionales" es la que más crece a lo largo del período, superando a la media nacional. A pesar de ello, lo que se destaca no son las bajas tasas de crecimiento relativo del rubro, sino el muy pobre nivel inicial del cual parten. En la tabla 9 pueden apreciarse los números que presenta la evolución de los profesionales y estudiantes a lo largo del período.

A pesar del gran crecimiento de la economía durante el período, los datos indican que Argentina no logró incrementar sustantivamente los números generales de profesionales en el mercado laboral. Tanto en los rubros profesionales como en el de estudiantes, en 1895 nos encontramos con una participación de los rubros en el mercado de trabajo llamativamente similar a la de 1870. Durante el segundo subperíodo, entre 1895 y 1913, el crecimiento de los profesionales se acelera desde una base muy pequeña, pero aun así es más bajo que el de los trabajadores semicalificados del sector industrial, lo cual demuestra que no es un sector que tenga gran pujanza en el crecimiento económico a escala global. En cuanto a la participación de los estudiantes en la población económicamente activa se puede plantear algo similar, aunque su crecimiento es más acelerado que el de los profesionales. 
TIEMPO \& ECONOMÍA Vol. 8 N.o 1 | Enero - Junio del 2021 pp. $11-4$

Tabla 9.

Profesionales y estudiantes en total y en porcentaje del total de trabajadores empleados en Argentina

Nota: la categoría "Profesionales" está compuesta por los profesionales de la salud, las ciencias, jurisprudencia y del sector mercantil. Debe aclararse que los individuos que continuaban estudiando mientras trabajan y son computados a otros empleos no son tenidos en cuenta en estos números. Fuente: elaboración propia con base en los censos nacionales de 1869,1895 y 1914.

\begin{tabular}{|c|c|c|c|c|c|c|}
\cline { 2 - 7 } \multicolumn{1}{c|}{} & $\mathbf{1 8 7 0}$ & $\mathbf{1 8 9 5}$ & $\mathbf{1 9 1 4}$ & $\begin{array}{c}\text { Participación } \\
\mathbf{1 8 7 0}\end{array}$ & $\begin{array}{c}\text { Participación } \\
\mathbf{1 8 9 5}\end{array}$ & $\begin{array}{c}\text { Participación } \\
\mathbf{1 9 1 4}\end{array}$ \\
\hline Profesionales & 3.315 & 12.452 & 35.137 & 0,39 & 0,76 & 1,09 \\
\hline Estudiantes & 2.855 & 8.295 & 43.348 & 0,33 & 0,50 & 1,34 \\
\hline $\begin{array}{c}\text { Total de } \\
\text { trabajadores }\end{array}$ & $\mathbf{8 5 3 . 0 4 3}$ & $\mathbf{1 . 6 4 5 . 6 4 0}$ & $\mathbf{3 . 2 3 3 . 2 5 3}$ & $\mathbf{1 0 0}$ & $\mathbf{1 0 0}$ & $\mathbf{1 0 0}$ \\
\hline
\end{tabular}

Tradicionalmente, se asocia una salida veloz de los niños del sistema educativo a factores inherentes a la oferta de empleo, como puede ser la expulsión de los niños y jóvenes de la escuela por una estrategia de supervivencia de las familias motivada por los bajos ingresos. Considero que esta hipótesis pierde vigencia si tenemos en cuenta que los salarios reales de la Argentina entre 1880 y 1900 se encontraban en un nivel relativamente elevado (Williamson, 1998, p. 49). En mi opinión, debe tenerse en cuenta la posibilidad de que este fracaso temprano del sistema educativo responda más a factores de la demanda de empleo, la cual no involucra profesiones de grandes saberes técnicos. Si consideramos, adicionalmente, que los salarios son elevados en el país, la hipótesis sobre la demanda podría acentuarse, puesto que estos altos ingresos que ofrece el mercado laboral significan para los estudiantes argentinos un enorme costo de oportunidad por seguirse educando y no tomar uno de los empleos bien pagados de mediana o baja calificación que la economía genera abundantemente.

Este costo de oportunidad, a su vez, se ve incrementado por las reducidas posibilidades de acceso a la educación superior en el país. En Argentina, hacia 1900 solo había universidades nacionales en la Capital Federal y la ciudad de Córdoba. A estas se les sumaría en 1905 la de La Plata, mientras que la federalización de la oferta educativa vendría tardíamente con la reforma universitaria de 1918 y la nacionalización de las universidades de Tucumán y del Litoral, fundadas solo unos pocos años antes. La escasa educación terciaria y su baja difusión por el vasto territorio nacional puede ser un factor relevante en la baja retención de jóvenes en el sistema educativo. Al observar los números de 1914, puede verse que luego de 1895 el reclutamiento de estudiantes fue aumentando. Es posible que al estar cambiando la estructura productiva, se demande mayor calificación de sus 
trabajadores. De cualquier manera, los números siguen marcando que el crecimiento en la educación superior de los jóvenes es modesto a lo largo de todo el período estudiado.

\section{CONCLUSIONES}

En este artículo, hemos intentado realizar algunas aproximaciones cuantitativas a la estructura ocupacional durante la Argentina de la primera globalización. A lo largo del período se destaca que la expansión del mercado laboral es enorme y que esta responde a la demanda dinámica de empleo que se crea en el país motivada por el elevado crecimiento de la economía. No obstante, también se plantea que este proceso muestra una muy alta heterogeneidad entre sectores y una lenta acumulación de calificaciones y destrezas. Las transformaciones de esta estructura laboral se consideran según los datos presentados y guardan similitudes con los antecedentes teóricos propuestos por Adam Smith y Simon Kuznets.

Se advirtió una cierta tendencia estable en la evolución de la estructura del mercado laboral entre 1870 y 1895, tanto en términos sectoriales como de calificaciones. Incluso, se advierte un incremento de la importancia del sector primario del mercado de trabajo durante este primer período, influida por la crisis de las industrias artesanales del interior. A nivel de calificaciones no se advierten grandes modificaciones y se mantiene una participación muy alta de los trabajadores agrícolas de baja calificación y con empleo estacional entre 1870 y 1895 . También hay indicios de cierto crecimiento de los propietarios rurales, a pesar de las defectuosas fuentes de 1869 , que son el resultado de la expansión de la frontera agrícola. Podemos advertir que el sector agropecuario, el de mayor dinamismo exportador en un marco de integración económica global, logró captar a una parte importante de la población económicamente activa, tanto entre los trabajadores como entre los propietarios. En términos de la teoría económica que se presentó en la introducción, podemos ahora decir que la evolución de la estructura se parece, en un primer momento, más a lo que predijo Smith. En este sentido, se destaca la gran importancia que tiene el sector primario en la captación y creación de empleos, el cual lidera el crecimiento de las exportaciones durante la primera globalización.

Entre 1895 y 1914, en cambio, la evolución de la estructura laboral parece ser diferente. Este es un período de emergencia de las industrias y comercios modernos, muchas veces relacionadas con los eslabonamientos 
de la dinámica economía exportadora. Puede verse este proceso en otros indicios, como la ampliación de la población urbana y la acumulación de calificaciones del grupo de los obreros y empleados, así como en la concentración de los capitales comerciales e industriales entre los propietarios.

Volviendo sobre los autores a los que hemos hecho referencia, podemos encontrar un crecimiento del empleo más similar a lo que plantea Kuznets en este segundo período. Esta teoría suponía que en un período largo de crecimiento existiría un proceso de cambio estructural liderado por sectores secundarios y terciarios modernos, que comienzan a demandar más empleo en el área urbana que en la rural. Así mismo, este proceso impulsa una evolución técnica de los sectores económicos que fomenta un incremento de los empleos de media y alta calificación respecto a los trabajadores no calificados. Efectivamente, eso se advierte desde 1895, aunque el viraje en las estructuras del empleo difícilmente pueda ser caracterizado como radical.

Teniendo en cuenta este cambio de matriz laboral, hemos podido advertir a lo largo del período que las tendencias modernizantes no son contundentes. El crecimiento del sector público (en administración y educación) —así como el stock de profesionales y el empleo urbano de mediana calificación- parece ser contundente, aunque no altera radicalmente el panorama general del mercado de trabajo. En el mismo, sigue predominando un trabajo de reducida calificación y de alta movilidad sectorial. Luego de un ciclo de largo y acelerado crecimiento económico en la Argentina de la primera globalización, estos cambios superficiales sobre la estructura laboral revelan que el proceso de acumulación de capacidades técnicas entre los trabajadores parece haber tenido un desempeño mucho menos impactante que el incremento del ingreso del país.

\section{AGRADECIMIENTOS}

El autor agradece los comentarios críticos de Ricardo Salvatore, María Camou, Fernando Rocchi, Camilo Martínez y demás panelistas de Southern Summer School 2018, Montevideo, Uruguay. 


\section{REFERENCIAS}

\section{Fuentes primarias}

Correa, A., \& Lahitte, E. (1898). Investigación parlamentaria sobre agricultura, ganadería, industrias derivadas y colonización. Taller Tip. de la Penitenciaría Nacional.

República Argentina (1872). Primer censo de la República Argentina: septiembre de 1869. Impr. del Porvenir.

República Argentina (1898). Segundo censo de la República Argentina: mayo 10 de 1895. Taller Tip. de la Penitenciaría Nacional.

República Argentina (1914). Tercer censo de la República Argentina: junio de 1914. Taller Tip. de la Penitenciaría Nacional.

\section{Referencias bibliográficas}

Allemandi, C. (2009). Una aproximación a las transformaciones económicas y ocupacionales de la ciudad de Buenos Aires y a las precariedades del torbellino modernizador. $V$ Jornadas de Jóvenes Investigadores. Instituto de Investigaciones Gino Germani, Universidad de Buenos Aires. https://www.aacademica.org/000-089/328

Allemandi, C. (2015). Sirvientes, criados y nodrizas: una aproximación a las condiciones de vida y de trabajo en la ciudad de Buenos Aires a partir del servicio doméstico (fines del siglo XIX-principios del XX). Siglo XXI.

Arceo, N., Fernández, A. L., \& González, M. (2019). El mercado laboral en el modelo agroexportador argentino: el rol de la inmigración. América Latina en la Historia Económica, 26(3), e952. https://doi.org/10.18232/ alhe.952

Barrancos, D. (2008). Mujeres, entre la casa y la plaza. Editorial Sudamericana.

Barrancos, D. (2012). Mujeres en la sociedad argentina: una historia de cinco siglos. Editorial Sudamericana.

Barsky, O., \& Gelman, J. (2001). Historia del agro argentino. Desde la Conquista hasta fines del siglo XX. Grupo editorial Grijalbo-Mondadori.

Beccaria, L. (2006). El Mercado de trabajo argentino en el largo plazo: el caso de la economía agroexportadora. CEPAL. https://doi.org/10.18356/ $7 \mathrm{~d} 85 \mathrm{e} 36 \mathrm{e}-\mathrm{es}$

Bértola, L., \& Ocampo, J. A. (2013). El desarrollo económico de América Latina desde la independencia. Fondo de Cultura Económica de México. 
Bragoni, B., \& Olguín, P. (2016). Salarios, precios y nivel de vida en Mendoza durante la transformación vitivinícola (1890-1914). Folia Histórica del Nordeste, 26, 156-177. http://dx.doi.org/10.30972/fhn.026893

Cortés-Conde, R. (1979). El progreso argentino, 1880-1914. Editorial Sudamericana.

Della-Paolera, G., \& Taylor, A. M. (2003). Tensando el ancla: La Caja de Conversión argentina y la búsqueda de la estabilidad macroeconómica, 18801935. Fondo de Cultura Económica de Argentina.

De-Vries, J. (2008). The industrious revolution: Consumer behavior and the household economy, 1650 to the present. Cambridge University Press. https://doi.org/10.1017/CBO9780511818196

Devoto, F. (2000). Historia de la inmigración. Editorial Sudamericana.

Díaz-Alejandro, C. (1970). Ensayos sobre la historia económica argentina. Amorrortu.

Ferrer, A. (2008). La economía argentina: desde sus orígenes hasta principios del siglo XXI. Fondo de Cultura económica.

Flores-Cruz, R. (2014). La discusión en torno a la migración y la movilidad territorial de la población. Universidad de Buenos Aires.

Gerchunoff, P., \& Llach, L. (2017). El ciclo de la ilusión y el desencanto: un siglo de políticas económicas argentinas. Emecé.

Germani, G., \& Graciarena, J. (1987). Estructura social de la Argentina: análisis estadístico. Editorial Raigal.

Kritz, E. (1985). La formación de la fuerza de trabajo en la Argentina, 18691914. Centro de Estudios de Población.

Kuznets, S. (1955). Economic growth and income inequality. The American Economic Review, 45, 1-28.

Llach, L. (2014). Newly rich, not modern yet. Mimeo.

Lobato, M. (2007). Historia de las trabajadoras en la Argentina (1869-1960). Edhasa.

Míguez, E. (2012). Historia económica de la Argentina: De la conquista a la crisis de 1930. Editorial Sudamericana.

Panettieri, J. (1990). Desocupación, subocupación, trabajo estacional y trabajo intermitente. De la crisis del 90 a la Primera Guerra Mundial. En J. Panettieri (ed.), Mercado de trabajo y paro forzoso (pp. 7-38). Editorial de la Universidad Nacional de La Plata. 
Pianetto, O. (1984). Mercado de trabajo y acción sindical en la Argentina, 1890-1922. Desarrollo Económico, 24(94), 297-307. https://doi. org/10.2307/3466742

Recchini, Z., \& Lattes, A. (1975). La población de Argentina. Ministerio de Economía de Argentina, Secretaría de Estado de Programación y Coordinación Económica, Instituto Nacional de Estadística y Censos.

Rocchi, F. (2000). Concentración de capital, concentración de mujeres. Industria y trabajo femenino en Buenos Aires 1890-1930. En F. Gil-Lozano, V. S. Pita y M. G. Ini (comps.), Historia de las mujeres en la argentina, Tomo II. Siglo XX.

Rocchi, F. (2005a). El péndulo de la riqueza: la economía Argentina en el período 1880-1916. En Z. Lobato (ed.), Nueva historia argentina: el progreso, la modernización y sus límites (1880-1916). Editorial Sudamericana.

Rocchi, F. (2005b). Chimneys in the desert: industrialization in Argentina during the export boom years, 1870-1930. Stanford University Press.

Sábato, H. (1989). Capitalismo y ganadería en Buenos Aires: la fiebre del lanar, 1850-1890. Editorial Sudamericana.

Smith, A. (1983). Investigación sobre la naturaleza y causa de la riqueza de las naciones. Hyspamerica.

Suriano, J. (2004). La cuestión social en Argentina, 1870-1943. Agencia Nacional de Promoción Científica y Tecnológica, Ministerio de Cultura y Educación, Secretaría de Ciencia y Técnica.

Suriano, J., \& Lobato, M. (2014). La sociedad del trabajo. Las instituciones laborales en Argentina durante la primera mitad del siglo XX. Edhasa.

Volkind, P. (2015). Entre la agricultura de punta y la canción de otoño: procesos de trabajo, medios de producción y relaciones sociales en los núcleos maiceros y trigueros bonaerenses, 1895-1920 (Tesis de doctorado, Universidad de Buenos Aires). Repositorio Universidad de Buenos Aires.

Williamson, J. (1998). Real wages and relative factor prices in the third world 1820-1940: Latin America. Harvard University Press. 


\section{ANEXO 1. CLASIFICACIÓN DE LOS EMPLEOS POR SEC- TORES Y EL NIVEL DE DESTREZAS A PARTIR DE LAS CATEGORÍAS CENSALES $(1869,1895$ Y 1914)}

En la siguiente sección, definimos los empleos que se tomaron por cada categoría delimitada. A continuación, se detalla cada uno de los oficios y trabajos delimitados entre los tres censos y se los ubica en su categoría general.

Trabajadores no calificados urbanos: aguadores, albañiles, amasadores, aserradores, canasteros, carboneros, calafates, cordeleros, costureras, escoberos, graseros, hojalateros, horneros, jaboneros, panaderos, pintores, peluqueros, tacheros, talabarteros, yeseros, changadores, lustradores, curanderos y jornaleros urbanos.

Trabajadores semicalificados y calificados urbanos: afinadores, afiladores, alpargateros, armeros, bronceros, caldereros, carpinteros, cerveceros, carniceros, chancheros, cigarreros, colchoneros, confiteros, constructores de buque, curtidores, doradores, empapeladoras, encuadernadores, fabricantes, fideeros, floristas, fundidores, gasistas, grabadores, herreros, trabajadores industriales diversos, joyeros, licoreros, litógrafos, maquinistas, marmoleros, mecánicos/electricistas, modistas, molineros, muebleros, paragüeros, pirotécnicos, plomeros, queseros, relojeros, sastres, sombrereros, tapiceros, tintoreros, tipógrafos, toneleros, torneros, veleros, vidrieros, plateros, cocheros, ferroviarios, telefonistas, telegrafistas, pedicuras, zapateros, bordadoras, ebanistas, hormeros y alfareros.

Trabajadores semicalificados y calificados rurales: peones, cazadores, mayordomos y alambradores.

Trabajadores no calificados rurales: leñadores, carreros, troperos, pastores, jornaleros rurales y mineros/canteristas.

Empleados y dependientes: agentes, empleados de comercio en general, corredores, cobradores, comisionistas, dependientes, empleados, repartidores, empleados de correos, vendedores y dependientes de abastecedores.

Productores agropecuarios pequeños y medianos: barraqueros, acopiadores, agricultores, horticultores, reconocedores de frutos y vinicultores.

Productores agropecuarios grandes: estancieros, hacendados y rentistas. 
Profesionales: tenedores de libros, abogados, escribanos, contadores, procuradores, dentistas, enfermeros, farmacéuticos, ortopédicos, médicos, parteras, químicos, veterinarios, arquitectos, dibujantes, agrimensores, calígrafos e ingenieros.

Educadores y técnicos: maestros, estudiantes, fotógrafos, escultores, artistas, literatos, periodistas, taquígrafos, traductores, músicos y acróbatas.

Propietarios comerciales e industriales: fondistas y hoteleros, propietarios de industrias en general, lecheros, banqueros, propietarios de comercio en general, empresarios y rematadores.

Militares y marinos: buzos, marinos, prácticos, pilotos y militares.

Servicio doméstico: caballerizos, cocineros, domésticos, trabajos domésticos, lavanderas y planchadoras.

Empleados estatales civiles: empleados públicos.

Cultos: clérigos no católicos, religiosas, sacerdotes, sacristanes y similares. 


\section{ANEXO 2. SELECCIÓN DE SECTORES ECONÓMICOS POR CATEGORÍAS OCUPACIONALES CENSALES (1870, 1895 Y 1914)}

Sector agropecuario: peones, agricultores, barraqueros, cazadores, estancieros, hacendados, horticultores, leñadores, obrajeros, mayordomos/capataces, cuidadores, pastores, reconocedores de frutos, vinicultores, alambradores, mineros, carreros, troperos, rentistas y jornaleros rurales.

Sector industrial-manufacturero: propietarios, afiladores, albañiles, alfareros, alpargateros, amasadores, aserradores, bordadoras, bronceros, caldereros, canasteros, carboneros, carpinteros, cerveceros, colchoneros, constructores de buque, cordeleros, costureras, curtidores, doradores, ebanistas, encuadernadores, escoberos, fideeros, fundidores, gasistas, grabadores, graseros, herreros, hojalateros, hormeros, horneros, industriales diversos (trabajadores), jaboneros, licoreros (empleados), litógrafos, maquinistas, marmoleros, mecánicos/electricistas, modistas, molineros (empleados), muebleros, panaderos, paragüeros, pirotécnicos, plomeros, relojeros, sastres, sombrereros, tacheros, talabarteros, tapiceros, tintoreros, toneleros, torneros, veleros, vidrieros, yeseros, zapateros y jornaleros urbanos.

Sector comercial-terciario: propietarios, abastecedores, acopiadores, afinadores, aguadores, armeros, calafates, carniceros, chancheros, cigarreros, confiteros, empapeladores, floristas, joyeros, pintores, peluqueros, queseros, tipógrafos, agentes financieros, corredores, cobradores, comisionistas, dependientes, empleados, rematadores, repartidores, cocheros, correos, changadores, telefonistas, telegrafistas, caballerizos, cocineros, lavanderos, lustradores, planchadores, trabajos domésticos, bañistas, pedicuras, taquígrafos, traductores/cartógrafos, acróbatas/deportistas, músicos y vendedores.

Sector público: buzos, ferroviarios, marinos, prácticos, pilotos, militares, empleados públicos, clérigos no católicos, religiosas, sacerdotes, sacristanes y maestros.

Profesionales y estudiantes: estudiantes, tenedores de libros, abogados, escribanos, contadores, procuradores, dentistas, enfermeros, farmacéuticos, ortopédicos, médicos, parteras, químicos, veterinarios, arquitectos, dibujantes, agrimensores, calígrafos, ingenieros, fotógrafos, escultores, artistas, literatos y periodistas. 